\title{
Production Planning and Inventory Control in a Remanufacturing Production System
}

\author{
Swee S. Kuik*, Toshiya Kaihara, Nobutada Fujii, Daisuke Kokuryo \\ Graudate School of System Informatics, Kobe University, 1-1 Rokkodai, Nada, Kobe, Hyogo 657-8501, Japan \\ *Corresponding Author: Swee.Kuik@ outlook.com
}

\begin{abstract}
The management of production and inventory control associated activities in remanufacturing production system has received attention by practitioners in the last two decades. This is due to the increased productivity, limited supply of virgin materials, stringent environmental requirements for product disposal and treatment as well as substatial change in global sustainability regulations. In order to constantly attain the supply chain cost savings for manufacturing operations, an integrated production planning model in remanufacturing by considering component obsolescence is developed. The aim of this developed model is to minimise an overall costs for production with remanufacturing operations. A numerical application is demonstrated to show its practical usefulness and flexibility in a remanufacturing production plan by considering different obsolete time periods for used components from returns streams. Finally, the contributions of this study and future works are discussed.
\end{abstract}

Keywords: Remanufacturing operations, reverse supply chain management, sustainable manufacturing, product recovery, component obsolescence.

\section{Introduction}

Supply chains is commonnly known as logsitical system that is to plan and organise the procurement of raw materials for manufacturing and distributing to their final consumers. [1-3] To reduce the used product landfill potential, manufacturers are now considering various remanufacturing operations as parts of their strategic planning towards sustainability [4-8]. Staiko et al [9] emphasised that manufacturers must be responsible for managing product returns and reovery operations. Currently, there is lacking on the appropriate production planing for the consideration of component obsolescence $[1,2,4,5]$. Furthermore, the production and inventory with remanufactured components is very difficult to manage due to the large variation of technological lifecycle and consumer demands. In particular, some used component may not able to be remanufactured for other secondary markets [10-12]. In this research study, we consider the complex scenario of production planning and inventory control with remanufacturing operations. A new optimisation model in remanufacturing is develeped by analysing and utilising used components for production and minimising disposal related treatment costs in a supply chain.

In addition, there is also a lack of discussion and review on the remanufactured component obsolescence scenario where the returned products and components are to be disassembled and utilised [10, 11, 13-15]. In this article, we discuss remanufacturing production planning and inventory control by varying used component obsolescence. Section 2 discusses the reverse supply chain management and product recovery operations. A gap in current literature for production planning in remanfuacturing is presented. In Section 3, the mathematical formulation is then presented and objective function is formulated for this study. Section 4 presents the numerical application to show the pratical usefulness of the proposed model. In Section 5, we present the results and analysis based on varying used component obsolescence time periods. A comparative study is analysed and discussed. Finally, the significant contributions and our future works are also discussed.

\section{Product Recovery}

\subsection{End of Life strategies}

To increase global competitiveness among supply chain networks, the considerations of end-of-life (EOL) strategies has recently become an alternative solution to generate 
additional profits for manfuacturers [16, 17]. Prices for acquiring vrigin materials for production are now getting higher and the environmental regulations has been revised regularly to meet current market needs [2, 4]. Therefore, there is an increase of total associated costs for used product disposal and treatment upon product returns.

The tradisional supply chain always aims for optimising the operational costs effectiveness and improving product order fulfilment and distribution to their end users. In reverse supply chain management, the obsolescence issue is one of the critical aspects for manufacturers to be considered for recovery operations as parts of their business processes [68]. This is due to the rapid change in technology lifecycle in which the used components may need to be disposed partly and/or entirely and unable to disassemble for other purpose or remanufacturing processes.

Althought there is an increased number of research literature that focuses on the importance of component obsolescnece and technology lifecycle in remanfuacturing, current optimisation modelling for production planning with obsolescence is still lacking due to complexity of product returns and recovery operations.

\subsection{Remanfuacturing operations}

In views of today's short lifecycle of new and innovative products, the remanufacturing strategy is now considered as one of the compromising ways to minimise large amount of product disposal for landfill $[2,3,5]$. This has become an important research focus to help manufacturers to cope with the higher costs of waste treamenet and increased burdens. There are various important factors and aspects that need to be examined for the technical feasibilities and lifecycle constraints including technology life and wear-out life [1-4]. Currently, there are numerous optimisation models for production planing for remanfuactruing operations but those models exclude the component obsoscelence issues. In fact, this is one of the major bottlenecks for manfuacturers to overcome this constraint $[10,17]$.

In today's markets, there are many remanufactured products that are currently available for consumers including single-use camera, mechanical machinery with components, laptop, automotive rebuilt parts and/or components [16, 17]. In addition, for achieving full financial sustainability, global manufacturers are also now working more on product returns and reovery operations improvement including production planning and inventory control to increase profitability of the organisation.

\subsection{Product Returns Management}

Kuik et al. [2, 4] also emphasised on the importance of improving product returns and remanfuacturing operations as manufacturers can generate significant cost savings by implementing the effective and efficient hanlding processes. For example, the returned product can be disassembled into multiple subassemblies for secondary markets and for other remanfuacturing processes $[2,3]$.

In recent years, the used component obsolescence issue is one of the upmost problems in recovery operations $[2,6]$. For effective production planning in remanfuacturing operations, the reduction of operational improvement programmes by minimising the inventory holding costs of those obsolete used components can actually improve the profitability of the organisation. Therefore, there is a need for developing integrated optimisation model for production planning in remanufacturing by incorporating obsolesence issue and minimising the total associated costs for production and inventory control.

\section{Mathematical Modelling}

The optimisation model is developed to examine various remanufacturing cost effective management in production planning. The developed model aims to minimise an overall associated costs for the remanufacturing operations and inventory holding including the collection returned costs.

Table 1. Model parameters

\begin{tabular}{cl}
\hline \hline & \multicolumn{1}{c}{ Description } \\
\hline$N$ & Number of remanufactured product $i$, where $i=1,2,3, \ldots N$ \\
$T$ & Number of component $j$, where $j=1,2,3, \ldots J$ \\
& Planning horizon comprising of time period $t$, where $t=$ \\
& $1,2,3, \ldots T$ \\
$(R+Q$ & Time period $f$, upon receiving component $j$, \\
$C_{j}$ & Unit cost of returned and remanufactured product $i$ \\
$U_{i}$ & Init cost of component $j$ \\
$H_{j}$ & Inventory holding unit cost for remanufactured product $i$ \\
$A_{i}$ & Fixed set-up cost for remanufactured product $i$ \\
$G_{j}$ & Fixed ordering cost for component $j$ \\
$V_{i}$ & Production capacity for remanufactured product $i$ \\
$M_{j}$ & Ordering capacity for component $j$ \\
$\alpha_{j}$ & Disposal treatment unit cost for component $j$ \\
$S_{j}$ & Order lot size ordering for component $j$ \\
$O_{j}$ & Order lead-time for component $j$ \\
\hline &
\end{tabular}


The detailed formation for this optimisation model is presented in the followsing section. The model paramenters and decision variables used for this formulation is tabulated in Table 1 and 2. The assumption made in this study is summarised as below:

- Demands are determinstics;

- Remanfuactured product and components are known;

- Remanfuacturing capacity and ordering are determinstic and constant;

- Reliability and quality aspects are excluded.

Table 2. Model variables

\begin{tabular}{cl}
\hline \hline & \multicolumn{1}{c}{ Description } \\
\hline$X_{i t}$ & Quantity of $i$ produced in t period \\
$I_{i t}$ & Quantity of inventory $i$ in t period \\
$y_{j t}$ & Quantity of $j$ ordered in t period \\
$L_{j t}$ & Quantity of inventory $j$ in t period \\
$L_{j t f}$ & Quantity of inventory in t period upon receiving $j$ at \\
& the end of f period \\
$\beta_{j}$ & Quantity of disposal treatment $j$ \\
$\gamma_{j t f}$ & Quantity of usage in t period upon receiving $j$ at the \\
& end of f period \\
$D_{i t}$ & Demand $i$ in t period \\
$B_{j i}$ & Bill of Material (BOM) with $j$ of $i$ \\
$e_{j}$ & Obsolescence with $j$ lifecycle \\
$a_{i t}$ & Fixed setup-up of $i$ in t period (binary) \\
$P_{j t}$ & Fixed (scheduled) ordering of $j$ in t period (binary) \\
$p_{j t}$ & Fixed ordering of $j$ in t period (binary) \\
&
\end{tabular}

\subsection{MIP Model Development}

This section discusses the mathematical formulation for a MIP optimisation model to examine remanufactured production planning scenario by considering remanufactured component obsolescence issue.

Using the above derived model parameters, an objective function is then formulated based on the costs associated with production planning and inventory control in a remanfuacturing production system as shown in Eq. (1). Term 1-4 are the primary cost associated with producing a remanufactured product with used components in production and inventroy. Term 5 is the production setup-up associated costs in time period planning horizon. Terms 6 and 7 are related to the cost associated with ordering used components for production. Term 8 is known as the inventory cost associated with used components in production planing at the end of obsolete time period. Finally, term 9 is used to estimate the disposal cost needed for obsolete inventory holding in production.

$$
\begin{aligned}
\operatorname{Min} Z=\sum_{i} \sum_{t}(R & +Q)_{i} X_{i t} \\
& +\sum_{j} \sum_{t} C_{j} y_{j t} \\
& +\sum_{j} \sum_{t} C_{j} Y_{j t}+\sum_{i} \sum_{t} U_{i} I_{i t} \\
& +\sum_{i} \sum_{t} A_{i} a_{i t}+\sum_{i} \sum_{t} R R_{j} P_{j t} \\
& +\sum_{j} \sum_{t} G_{j} p_{j t} \\
& ++\sum_{j} \sum_{t} \sum_{f} H_{j} L_{j t f}+\sum_{j} \alpha_{j} \beta_{j}
\end{aligned}
$$

The following specifc contraints are used for deriving the objective function.

$$
\begin{aligned}
& I_{i t}=I_{i, t-1}+X_{i t}-D_{i t}, \forall t, j \\
& \sum_{f}^{t} L_{j t f}=L_{j 0}+S_{j} Y_{j t}-\sum_{i} B_{j i} X_{i t}, t=1, \forall t, j, r \\
& \sum_{f}^{t} L_{j t f}=\sum_{f}^{t-1} L_{j, t-1, f}+S_{j} Y_{j t}-\sum_{i} B_{j i} X_{i t}
\end{aligned}
$$$$
1<t<e_{j}, t \geq e_{j}, \forall t, j
$$

$\sum_{f=t+1-e_{j}}^{t} L_{j t f}=\sum_{f=t+1-e_{j}}^{t-1} L_{j, t-1, f}+S_{j} Y_{j t}-\sum_{i} B_{j i} X_{i t}$

$$
t \geq e_{j}, t \geq o_{j}, \forall t, j
$$

$\sum_{f}^{t} L_{j t f}=\sum_{f}^{t-1} L_{j, t-1, f}+S_{j} y_{j t}-\sum_{i} B_{j i} X_{i t}$

$$
1<t<e_{j}, t \geq o_{j}, \forall t, j
$$

$\sum_{f=t+1-e_{j}}^{t} L_{j t f}=\sum_{f=t+1-e_{j}}^{t-1} L_{j, t-1, f}+S_{j} y_{j t}-\sum_{i} B_{j i} X_{i t}$,

$\sum_{i} B_{j i} X_{i t}=\sum_{f} \gamma_{j f \mathrm{t}}$

$$
t \geq e_{j}, t \geq o_{j}, \forall t, j
$$

$\sum_{i} B_{j i} X_{i t}=\sum_{f=t+1-e_{j}} \gamma_{j f t}$ $1 \leq t<e_{j}, \forall t, j, r$

$L_{j t f}=S_{j} Y_{j t}-\gamma_{j f t}$ $e_{j} \leq t<T, \forall t, j, r$ 


$$
f=t, t \leq o_{j}, \forall t, j, r
$$

$L_{j t f}=S_{j} y_{j t}-\gamma_{j f t}$

$$
f=t, t \leq o_{j}, \forall t, j, r
$$

$L_{j t f}=L_{j, t-1, f}-\gamma_{j f t}$

$f=t, t \leq o_{j}, \forall t, j, r$

$\beta_{j}=\sum_{t=o_{j}-1} \gamma_{j, t, t+1-o_{j}}$

$X_{i t} \leq V_{i}, y_{j t} \leq M_{j}$

$y_{j t} \leq W P_{j t}, X_{i t} \leq W a_{i t}, Y_{j t} \leq W p_{j t}$ $\forall t, j$

$X_{i t}, y_{j t}, Y_{j t}, I_{i t}, L_{j t f}, \gamma_{j f t}, \beta_{j} \geq 0$

$\forall t, i$

$a_{i t}, P_{j t}, p_{j t} \in(0,1)$

$$
\forall t, i, j, f
$$

Eq. (2) is considered as the balance of inventory holding in production that must be satisfied with the demand request. Eqs (3) - (7) are classified as each remanufacturing order is corresponding with the scheduled production. Eqs. (8) and (9) is related to the remanufacturing production usage at each period with the obsolete used components. Eqs. (10) - (12) are used for inventory control for product and used component at each period. Eq. (13) is used for the excessive components to be disposed at the end of its obsolete period. Furthermore, the remanufacturing capacity constraints are formulated as shown in Eq. (14). Eq. (15) is known as the decision variable in binary form. Under the condition, these variables of $y_{j t}, P_{j t}$, and $p_{j t}$ are known as one, where $W$ is represented as a large positive number. Finally, Eqs. (16) and (17) are the continuous and binary decision variables used in study. For solving this optimization model with obsolete used component scenario as shown in Eqs. (1), a CPLEX mixed integer optimization solver is used.

\section{Numerical Example}

This section discusses the numerical example used in remaufacturing production system. In this study, we set the planning horizon of 12 periods. In this model, there are three types of remanufactured products (i.e. RP1, RP2, and RP3) to be assembled by four separate components (i.e. CP1, CP2, CP3, CP4). These separate components have different timeperiod for obsolescence from returned collection. In the following, Table 3 shows the important parameters used for remaufactured product in the modeling. Table 4 shows that the related parameters for used components in the modeling.
Table 3. Parameters used product $i$ for modelling

\begin{tabular}{|c|c|c|c|}
\hline & PT1 & PT2 & PT3 \\
\hline$V_{i}$ & 300 & 700 & 700 \\
\hline$(R+Q)_{i}$ & 115 & 100 & 230 \\
\hline$U_{i}$ & 56 & 48 & 77 \\
\hline$A_{i}$ & 530 & 780 & 840 \\
\hline
\end{tabular}

Table 4. Parameters used component $j$ for modelling

\begin{tabular}{|c|c|c|c|c|}
\hline & $\boldsymbol{C P 1}$ & $\boldsymbol{C P 2}$ & $\boldsymbol{C P 3}$ & $\boldsymbol{C P 4}$ \\
\hline$S_{j}$ & 4500 & 4500 & 10000 & 10000 \\
\hline$M_{j}$ & 60 & 30 & 6 & 8 \\
\hline$C_{j}$ & 7500 & 22000 & 35000 & 23000 \\
\hline$L_{j t}$ & 4 & 5 & 5 & 3 \\
\hline$\alpha_{j}$ & 6 & 8 & 9 & 6 \\
\hline$G_{j}$ & 420 & 540 & 680 & 460 \\
\hline$O_{j}$ & 30000 & 22000 & 5000 & 0 \\
\hline
\end{tabular}

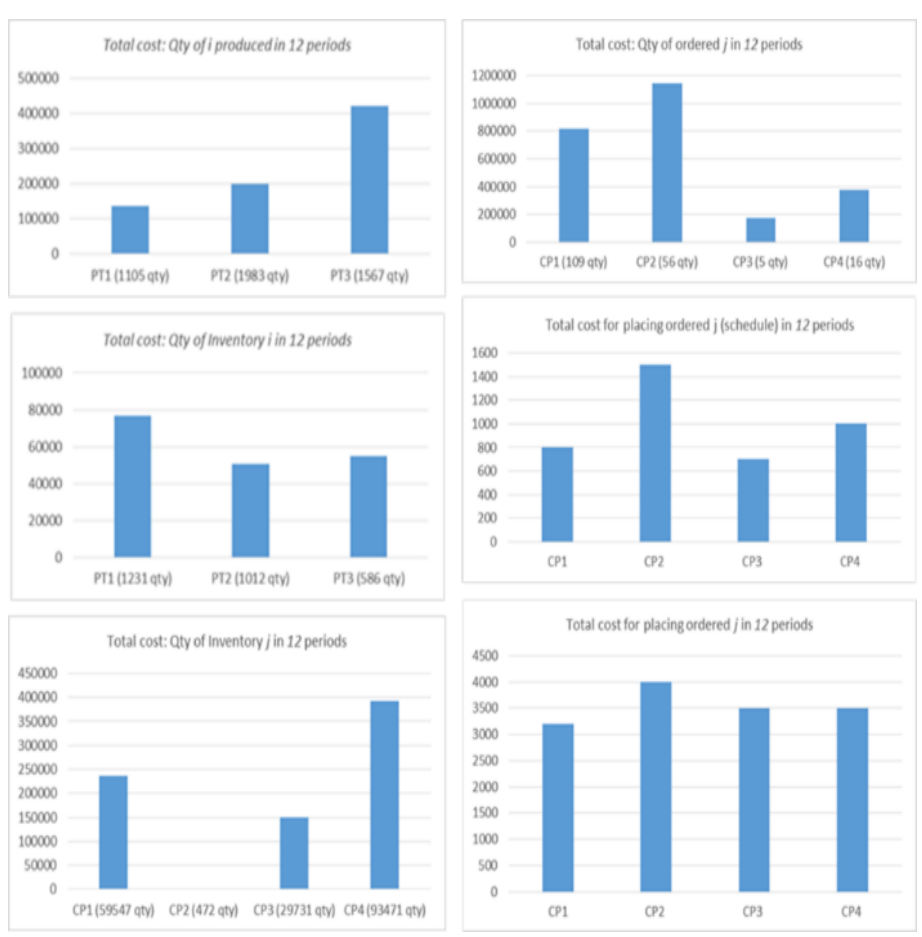

Fig. 1. A summary of total associated costs for producing 3 product types with 4 components

In this study, we consider 6 months for CP1, 3 months for CP2, 4 months for CP3, 3 months for CP4. We develop the optimisation model and solve it using Cplex optimisation solver. In addition, in this study, we also consider the practical scenario by considering the increase of $5 \%, 10 \%$ and $15 \%$ of obsolescence time-period for each separate components. Results obtained showed an overall minimum 
costs for remanufacturing production planning was approximately $\$ 6,029,983$ with linear relaxation of approximately $\$ 5,528,876$.

\section{Discussion}

Due to rapid change in technological development, many reused components are used for a new manufuctured products. Furthermore, as mentioned earlier, the product obsolescence management is one of the important issue in remanufacturing operations. In this study, we also compare various obsolete time-period scenario for used components from returned collection. The obtained results revealed that total associated costs in remanufacturing is significantly reduced as illustrated in Figure 2.

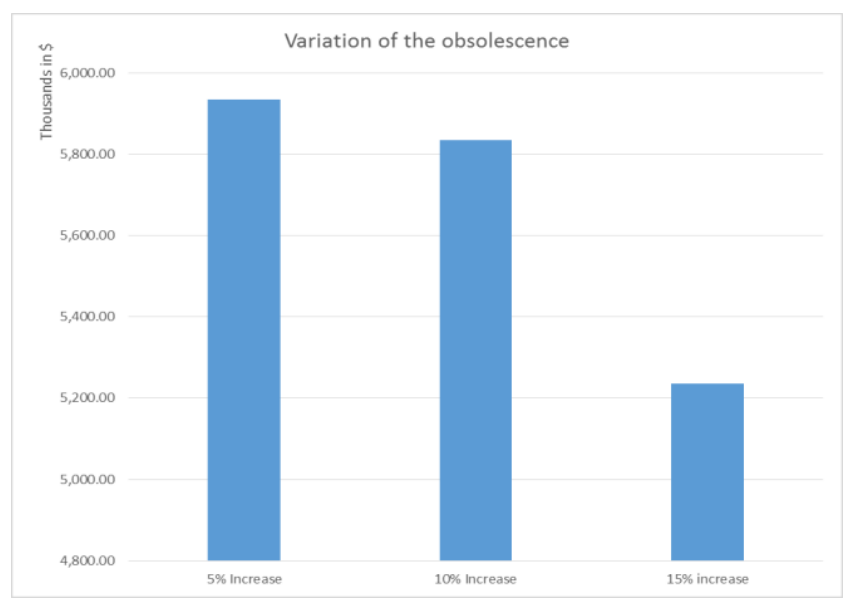

Fig. 2. A summary of variation of the obsolescence

Figure 2 illustrates clearly that when there is an increase of $5 \%, 10 \%$ and $15 \%$ in terms of the obsolete time-period used components, total associated costs in remanfuacturing operations is reduced dramatically. The is primarily due to the significant reduction of production and inventory holding costs for those obsolete used components in production. This is implied that if technology lifecycle for a remanufactured product is further extended and longer, the remanufacturing costs for producing a remanufactured product is significantly reduced as well. This study proved that the effect of varying obsolete time period for used component is also one of the crucial factors in remanufacturing production operations.

\section{Conclusion}

The proposed optimisation model for production planning has been developed to take into consideration of the component obsolescenec for a remanufacturing production system. Comparisons of the ontained results for used component obsolescence shows that the associated costs for producing a remanufactured product is significantly reduced if the obsolete time of the used components from returned collection is increased from $5 \%$ to $15 \%$.

In addition, the contribution of this study is twofolds. Firstly, this optimisation model aims to analyse and examine used component obsolescence issue for production planning and inventroy control in remanufacturing from returned collection. Secondly, the proposed optimisation model by incorparating the obsolescence element is developed to tackle and resolve some oversimplifications in numerical optimisation modelling and analysis. For our future works, we also plan to consider the uncertainty of product returns and recovery in reverse supply chain.

\section{References}

(1). Swee Kuik, Toshiya Kaihara, Nobutda Fujii and Daisuke Kokuryo, An Integrated Production Planning Model with Obsolescence and Lifecycle Considerations in a Reverse Supply Chain, in Advances in Production Management Systems: Innovative Production Management Towards Sustainable Growth, S. Umeda, et al., Editors., Springer International Publishing. p. 680-688, 2015

(2). Swee Kuik, Sev Nagalingam and Yousef Amer Sustainable supply chain for collaborative manufacturing. Journal of Manufacturing Technology Management, 22(8): p. 984-1001, 2011

(3). Swee Kuik, Sev Nagalingam and Yousef Amer. Assessing Sustainability in Manufacturing Using a Fuzzy Expert System. in International Conference on Innovations in Bio-Inspired Computing and Applications (IBICA), 2012.

(4). Swee Kuik, Toshiya Kaihara, and Nobutada Fujii. Stochastic Decision Model of the Remanufactured Product with Warranty. in Lecture Notes in Engineering and Computer Science: Proceedings of the International MultiConference of Engineers and Computer Scientists. Hong Kong, 2015

(5). Swee Kuik, Sev Nagalaingam and Yousef Amer. Challenges in implementing sustainable supply chain within a collaborative manufacturing network. in Supply Chain Management and Information Systems (SCMIS), 2010 8th International Conference on. 2010.

(6). Sev Nagalingam, Swee Kuik and Yousef Amer, 
Performance measurement of product returns with recovery for sustainable manufacturing. Robotics and Computer-Integrated Manufacturing, 29(6): p. 473483., 2013

(7). Jiali Zhu, Toshiya Kaihara, Nobutada Fujii, Daisuke Kokuryo and Swee Kuik, Extended EOQ model considering demand fluctuation in reverse logistics. in Society of Instrument and Control Engineers of Japan (SICE), 2015 54th Annual Conference of the. 2015.

(8). Hao Lin, Sev Nagalingam, Swee Kuik, Tomohiro Murata, Design of a Global Decision Support System for a manufacturing SME: Towards participating in Collaborative Manufacturing. International Journal of Production Economics, 136(1): p. 1-12, 2012

(9). Theodoros Staikos and S. Rahimifard, An end-of-life decision support tool for product recovery considerations in the footwear industry. International Journal of Computer Integrated Manufacturing, 2007. 20(6): p. 602-615, 2007

(10). Mehmet Ilgin and Surendra Gupta, Environmentally conscious manufacturing and product recovery (ECMPRO): A review of the state of the art. Journal of Environmental Management, 91(3): p. 563-591, 2010

(11). Kai Jin and Hong Zhang, A decision support model based on a reference point method for end-of-life electronic product management. International Journal of Advanced Manufacturing Technology, 31(11/12): p. 1251-1259, 2007

(12). Michael R. Johnson and Ian McCarthy, Product recovery decisions within the context of Extended Producer Responsibility. Journal of Engineering and Technology Management, 34(0): p. 9-28., 2014

(13). Vlachos, D., P. Georgiadis, and E. Iakovou, A system dynamics model for dynamic capacity planning of remanufacturing in closed-loop supply chains. Computers \& Operations Research, 34(2): p. 367-394, 2007

(14). Iakovou E et. al, Disassembly planning and sequencing for end-of-life products with RFID enriched information. Robotics and Computer-Integrated Manufacturing, 29(3): p. 112-118, 2013

(15). Yamada, T. and Ohta T., Modeling and Design for Reuse Inverse Manufacturing Systems with Product Recovery Values, in Advances in Sustainable Manufacturing, G. Seliger, M.M.K. Khraisheh, and I.S. Jawahir, Editors. Springer Berlin Heidelberg. p. 197202, 2011

(16). Marcus Brandenburg, Kannan Govinda, Joseph Sarkis and Sefan Seuring, Quantitative models for sustainable supply chain management: Developments and directions. European Journal of Operational Research, 233(2): p. 299-312, 2014

(17). Debabrata Das and Pankaj Dutta, A system dynamics framework for integrated reverse supply chain with three way recovery and product exchange policy. Computers \& Industrial Engineering, 66(4): p. 720-733, 2013 